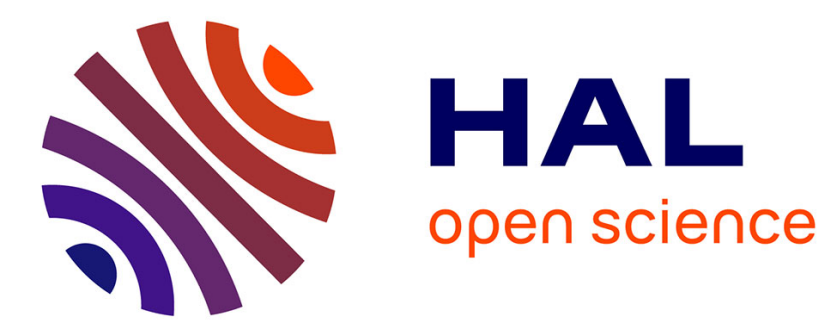

\title{
SN démonstratifs "prédicatifs": qu'est ce qui limite leur apport informatif
}

\author{
Catherine Schnedecker
}

\section{To cite this version:}

Catherine Schnedecker. SN démonstratifs "prédicatifs": qu'est ce qui limite leur apport informatif. Langue française, 2006, 152, pp.39-55. hal-00808798

\section{HAL Id: hal-00808798 \\ https://hal.science/hal-00808798}

Submitted on 7 Apr 2013

HAL is a multi-disciplinary open access archive for the deposit and dissemination of scientific research documents, whether they are published or not. The documents may come from teaching and research institutions in France or abroad, or from public or private research centers.
L'archive ouverte pluridisciplinaire HAL, est destinée au dépôt et à la diffusion de documents scientifiques de niveau recherche, publiés ou non, émanant des établissements d'enseignement et de recherche français ou étrangers, des laboratoires publics ou privés. 


\section{SN démonstratifs "prédicatifs " : qu'est-ce qui limite leur apport informatif ?}

\section{INTRODUCTION 1}

Notre étude portera sur le type d'anaphore démonstrative particulier qu'illustrent les énoncés (1) à (5) :

1) Un chasseur est arrivé hier; cet Allemand a manqué tous ses tirs (J.C. Milner, 1982, 24, ex. 7a)

2) Il y avait sur la table un livre. Ce roman... (F. Corblin, 1987, 210)

3) Il y avait sur la table un roman. Ce livre... (G. Kleiber, 1988, 65)

4) Il y avait sur la table un livre. Ce torchon... (F. Corblin, 1987, 210)

5) Mais voilà qu'un beau matin, une vendeuse les sortit de leur boîte pour les essayer à une dame. La dame/l'aimable cliente/cette dame/cette bourgeoise mal attifée/elle les mit, fit quelques pas avec... (M.-J. ReichlerBéguelin, 1995, 54)

Ces SN démonstratifs s'y caractérisent en ce que leur tête lexicale apporte, sur le référent, des informations inédites, qu'on ne peut inférer ni du segmentsource, ni, le cas échéant ( $c f .1)$, du cotexte inséré entre les deux expressions référentielles. En ce sens, elles équivalent à une prédication. C'est pourquoi nous les qualifierons désormais de " $\mathrm{SN}$ démonstratifs prédicatifs » (en abrégé : $\mathrm{SNDP})$. Bien que le phénomène ne concerne pas exclusivement les démonstratifs $^{2}$, nous limiterons notre étude à ces formes. En effet, les SNDP posent toute

1. Nous remercions chaleureusement G. Kleiber et W. De Mulder pour leur relecture de ce texte ainsi que pour leurs observations et suggestions comme toujours stimulantes.

2. On rencontre en effet ce genre de problème avec les SN définis : Lors d'une soirée au cours de laquelle alcool et cannabis sont consommés, Julien se rend à un rendez-vous fixé par un dealer. Lorsque ce dernier est retrouvé mort, l'adolescent est accusé de meurtre et finit par avouer. (TV Hebdo, 13/10/ 2004). 
une série de problèmes. Le premier - et non des moindres - tient à ce qu'ils dérogent aux mécanismes anaphoriques coréférentiels "standards ", décrits généralement comme un rappel d'informations, préalablement encodées ou déductibles par inférence d'unités du cotexte antérieur, ainsi que le rappellent les trois extraits de définition (i) à (iii) :

i) Il y a relation d'anaphore entre deux unités $A$ et $B$ quand l'interprétation de $B$ dépend crucialement de l'existence de $A$, au point qu'on peut dire que l'unité $B$ $n^{\prime}$ est interprétable que dans la mesure où elle reprend - entièrement ou partiellement - A. (J.-C. Milner, 1982, 18)

ii) Il me semble ainsi que le plus souvent, l'anaphore se ramène pour l'essentiel à un simple passage à l'hyperonyme (c'est en cela qu'on peut y voir, par rapport à la répétition intégrale, un procédé d'économie) (...) (A. Berrendonner, 1983, 236)

iii) L'anaphore se caractérise (...) comme un phénomène de rappel informationnel relativement complexe où sont susceptibles d'intervenir :

1) le savoir construit linguistiquement par le texte lui-même ;

2) les contenus inférentiels qu'il est possible de calculer à partir des contenus linguistiques pris pour prémisses, et cela grâce aux connaissances lexicales, aux prérequis encyclopédiques et culturels, aux lieux communs argumentatifs ambiants dans une société donnée. (M.-J. Reichler-Beguelin, 1988, 18, souligné dans le texte).

Le second problème est lié à la manière d'évaluer la nouveauté informationnelle. Tout dépend, en effet, de ce que l'on se donne comme point de repère. Lorsque celui-ci se confond avec le SN-source comme dans les exemples (1) à (5), le SND est effectivement " prédicatif ». Encore faut-il s'assurer qu'il ne tire pas sa substance du contenu propositionnel qui le précède, comme cela se produit en (6) et (7) où ce jardinier et cette unique passagère sont générés respectivement à partir des SV être maraîcher et se retrouver seul à bord d'un Boeing 747 :

6) M. Soubeyrou est maraîcher des environs.

Trois fois par semaine, mon père donne quelques leçons au fils de ce jardinier (...). (J. Vallès, in M.-J. Reichler-Beguelin, 1995, 62)

7) Une Japonaise a pu choisir la meilleure des 353 places d'un Jumbo, s'étant retrouvée seule à bord d'un Boeing 747, a annoncé British Airways. N'ayant pu la transférer sur un autre vol, la compagnie s'est retrouvée obligée de maintenir le vol pour cette unique passagère. (La Suisse, M.J. Reichler-Beguelin, ibid.)

Dans ce cas, il n'y a pas prédication via le SND; il s'agit tout au plus d'une anaphore qui reprend et, d'une certaine façon, synthétise des contenus propositionnels : dans ce sens on aurait affaire à une anaphore « résomptive ${ }^{3}$.

Outre la question de leur point de repère, les SNDP rencontrent celle de $l^{\prime}$ « unité » textuelle de leur occurrence, au sens ici de M. Ariel et des approches

3. Le repérage de la source peut se révéler ambigu, comme le souligne M.-J. Reichler-Béguelin avec l'extrait: "Quelques camions faisaient des aller et retour entre la montagne et la ville. Mais ces navettes demeuraient évidemment dérisoires » (Le Monde, cité par M.-J. Reichler-Béguelin, 1995 : 60) qui se demande : [...] Faut-il y (= l'expression linguistique requise pour l'interprétation du rappel) inclure le sujet et tous les arguments du prédicat? Faut-il délimiter ainsi <cf. expressions en gras $>$ ou plutôt ainsi <nos italiques $>$ ? 
« hiérarchiques » de l'expression de la référence. En effet, le contenu du SN cet Autrichien de (8) n'est pas à proprement parler "nouveau », étant donné que l'information figure déjà dans le chapeau de l'article. Or, deux facteurs seraient susceptibles ici de désactiver celle-ci : la distance entre la mention des deux informations, d'une part, et surtout, leur occurrence dans deux unités paragraphiques et énonciatives distinctes. Dans cette perspective, l'information contenue dans cet Autrichien serait nouvelle et il y aurait effectivement prédication :

8) Hubert Sauper, un œil sur ce bas monde

Dans « Le Cauchemar de Darwin », le réalisateur autrichien utilise l'image comme argument pour dénoncer la mondialisation. Il maîtrise l'art de frapper en finesse, sans commentaire ni jugement.

Y a-t-il un fond au puits de l'abjection? À la question, Hubert Sauper répond caméra au poing. Ou plutôt avec une caméra en guise de poing. Pas à la manière d'un Michael Moore, dont les documentaires à thèse s'apparentent à des chemins d'uppercuts bien alignés d'où le spectateur ne peut guère s'échapper. Sauper frappe en finesse. Il a l'art de vous restituer son coup d'œil sur ce bas monde sans jugement ni colère. À vous de juger, à vous de vous fâcher.

Pourtant, rien ne semblait prédestiner cet Autrichien à devenir témoin de notre planète. Né au bas de la Streif, la mythique piste de ski de Kitzbühel, il est enfant d'hôteliers. Mais la station tyrolienne, bien que perdue dans les Alpes, est un concentré de cosmopolitisme: "J'ai grandi, dit-il, dans un lieu public, le restaurant de mes parents, au milieu d'Italiens, d'Allemands, de Slovaques... » Il prend des photos, semble suivre la trace familiale en effectuant des stages en hôtellerie, voyage, puis, à 22 ans, choisit la voie de l'image et $\mathrm{du}$ voyage. Premier documentaire sur des Tsiganes en Roumanie. (Le Monde, 02.03.05)

Un troisième problème est lié au contenu possible des SNDP : est-il totalement libre? i.e. est-il possible de prédiquer via les SND «tous azimuts » ? Ou l'apport informationnel est-il, au contraire, contraint, régulé et, le cas échéant, par quoi ${ }^{4}$ ? Les discordances dans les jugements de recevabilité auxquels ont prêté, par exemple, (2), (3) et (4) montrent que la question est cruciale. Ainsi G. Kleiber (1988, 58 et 65) les indexe-t-il. (1) et (5), quant à eux, nous semblent un peu difficiles.

C'est précisément ce troisième problème que nous traiterons, et ce, en trois temps. Dans un premier temps, nous énumérerons et classerons les moyens fort nombreux par lesquels il est possible de «prédiquer » via les SND. Nous passerons ensuite en revue très rapidement les opinions contradictoires face à ce phénomène, ce qui nous donnera l'occasion de rappeler les « freins » qui lui ont été posés. Une fois examinées leurs limites, nous ferons valoir deux contraintes supplémentaires : 1'une, inspirée par G. Kleiber (1990 et 2001), faisant intervenir le mode de donation défini vs indéfini de la source qui suppose que, dans le cas de l'indéfini, le cotexte doit être en mesure de fournir, d'une manière ou d'une

4. J.-C. Milner $(1982,25)$ soulève un quatrième problème en suggérant que les unités démonstratives ne sont pas anaphoriques. 
autre, la «source » des informations nouvelles. La seconde contrainte repose sur le principe de cohérence.

Deux précisions avant de commencer. Pour des raisons de commodité, nous limiterons cette étude à des séquences où l'information du SNDP est totalement nouvelle. Deuxièmement, nous travaillerons sur des extraits à référents humains principalement, provenant en partie d'un corpus de portraits journalistiques constitué "manuellement », ce genre semblant particulièrement prédisposé aux SNDP.

\section{CE QUI, DANS LE SN DÉM, PEUT APPORTER DU NOUVEAU}

Le fait de parler de SNDP ne doit pas masquer la diversité des moyens par lesquels est véhiculée l'information nouvelle. Nous commencerons par inventorier les procédés sur la base de typologies déjà proposées par P. Lerat (1988), H. Manuélian (2003) et M.-J. Reichler-Béguelin (1988, 1989, 1995a, b) que nous détaillerons ou compléterons, le cas échéant. Ces typologies s'appuient principalement sur deux critères : la catégorie des N-têtes (nom propre vs commun, nom nu vs expansé, etc.) et, si nécessaire, la relation sémantique qu'entretiennent, le cas échéant, le SND et sa "source ». Nous les présentons ici par ordre d'informativité croissante.

\section{I.I. Le(s) modifieur(s) du SN démonstratif à N hyperonyme}

Une première catégorie de SNDP ( $c f .9$ et 10) se compose d'un $N_{\text {hyperonyme }}$ modifié (cf. fille et homme). Ces séquences sont mixtes du point de vue informationnel car le $\mathrm{N}_{\text {hyperonyme }}$ appauvrit d'une certaine façon plus qu'il ne renouvelle l'information référentielle qui se trouve entièrement portée, du coup, par les expansions du $\mathrm{N}$ souvent nombreuses et disparates. Celles-ci sont d'ailleurs souvent le lieu où s'expriment les jugements de valeur du locuteur sur le référent :

9) Ce sont eux qui ont fait Hélène, guidant ses expériences initiales et lui donnant enfin un premier rôle, le personnage de Aïe dans le film du même nom, tourné par la frangine en 2000.

Peu à peu on s'est mis à remarquer cette grande fille à la beauté androgyne, jolie garce de comédie (...). (Libération, 09/12/03)

10) Il (J. Villeret) y incarnait François Pignon, l'humble fonctionnaire humilié puis triomphant, un rôle qui lui valut le César du meilleur acteur en 1999. Avec plus de neuf millions d'entrées, le film reste l'un des plus grands succès de l'histoire du cinéma français et a fixé durablement l'image de cet homme un peu trop gros (quoique sa silhouette ait souvent varié au fil des ans), aux grands yeux naïfs, si gentil qu'il parvient à surmonter la méchanceté du reste de l'humanité. Mais ce n'est pas rendre justice à cet acteur étonnant que de se limiter à cette version-là de l'acteur. (Le Monde, 02/02/05)

\section{I.2. La tête lexicale du SN dém}

Parfois, l'information est véhiculée par la seule tête lexicale du SND. On distingue alors deux cas de figure. 
- Le SNDP entretient avec sa source un rapport sémantique conventionnel : l'hyponymie (11) ou la para-synonymie (12) :

11) C'est aussi le cas de Behrens, qui, après avoir eu la douleur de perdre un petit enfant, prit le risque à 46 ans, d'en avoir un autre. Six ans plus tard, la présence de cette petite fille fait de Behrens une jeune mère à l'épanouissement physique spectaculaire (M.-J. Reichler-Béguelin, 1989, 314, son ex. 14)

12) Et tout cela se déroulait sur fond d'une confrontation directe entre $l^{\prime}$ Est et l'Ouest. Bien sûr, je me demandais s'il fallait continuer cet affrontement ou chercher d'autres possibilités (Le Monde, in M.-J. Reichler-Béguelin, 1995, 70, son ex. 14)

- Le SNDP est sans rapport sémantique avec sa source. Dès lors, nombreux sont les $\mathrm{N}$ susceptibles d'apporter un éclairage nouveau, notamment dans le cas des référents humains, dont ils éclairent les diverses facettes ${ }^{5}$. C'est ainsi qu'ils peuvent dénoter des informations «objectives », telles que la nationalité (13) (ou la religion), la profession/fonction (14) ou encore l'« état civil » (15 et 16) :

13) Il n'a pas la blondeur ni la verve insolente d'Eminem. Avec sa veste militaire, qu'un ami peintre a décorée d'un portrait de Gandhi, Lawrence Muggerud, alias DJ Muggs, prétendrait plutôt à une certaine sagesse.

Barbu aux épaules larges, cet Italo-Américain a préféré les platines au micro, l'ombre aux feux de la rampe. (Le Monde, 20/06/03)

14) Kendal Nezan, gardien

Le directeur de l'Institut kurde de Paris consacre tous ses moyens à la sauvegarde de la culture de son peuple, dispersé dans plusieurs pays. Il rentre d'un voyage au Kurdistan d'Irak et, pour lui, l'avenir se joue « làbas ", dans les changements en cours de la mémoire kurde.

Européen convaincu, Français d'adoption, Parisien par la force de l'habitude, Kendal Nezan est kurde. Le 24 février, il a fêté les vingt-deux ans d'existence de l'Institut kurde de Paris. Il en est l'âme, lui qui a fait du lieu - une modeste bâtisse plantée au fond d'une cour du $10^{\mathrm{e}}$ arrondissement de Paris - $\mathrm{l}^{\prime}$ « ambassade culturelle » de ce peuple moyen-oriental de 30 millions d'âmes dispersé entre la Turquie, la Syrie, l'Irak, l'Iran et l'ex-URSS.

Il reçoit dans son bureau dépouillé du premier étage. Regard bleu limpide, voix suave, ce physicien de formation rechigne à parler de lui : "Ma personne? Quel intérêt? Chez nous, on est pudique! A moins qu'il ne s'agisse de parler des Kurdes... » Sur le sujet, il est intarissable. (Le Monde, 25/02/05)

15) Vaidelotis le Letton

Architecte de formation, cet octogénaire a connu toutes les étapes tragiques de son pays, stalinisme, occupation nazie, absorption par l'ex-URSS. En sep-

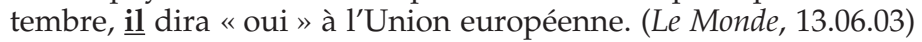

16) Benicio Del Toro n'a certes aucun signe extérieur du star system. Pas de villa cossue à Beverly Hills, mais toujours le même deux pièces de West Los Angeles où ce célibataire vit comme un étudiant, encombré de ses toiles de peintre amateur, de bouquins, et de vidéos de vieux films européens. (Libération, 16/01/04)

5. Ils remplissent également d'autres fonctions discursives mises en évidence par M.-J. ReichlerBéguelin (1995). 
Ils peuvent aussi renvoyer à diverses propriétés (physiques et/ou psychologiques) du référent et s'investir, le cas échéant, de l'opinion du locuteur (cf. 17 à 19) :

17) Comme quand quelqu'un commence à pérorer, à pontifier dans une conversation et vous vous regardez en réprimant votre irrésistible envie de rigoler devant cet abruti. (Corpus Leipzig, 1996)

18) Un modeste retraité désireux de convoler enfin en justes noces doit se faire passer pour beaucoup plus riche qu'il n'est afin de pouvoir conquérir sa dulcinée. Cette matrone demande alors au «bourgeois malgré lui » d'user de son pouvoir afin de venir en aide à sa belle-fille aveugle. (TV Obs, 15/11/04)

19) Malgré sa fraîcheur un peu naïve et plutôt bienvenue, il (= Stefano Pilati) n'ignore pas que si, par miracle, il sort la marque YSL de l'ornière, il lui faudra aussi assumer son exposition dans les médias. Ce grand timide, qui a longtemps rougi avant de se lancer dans une conversation, dit ne pas avoir d'idées sur la façon dont il modifierait alors son comportement. (Libération, 31 janvier 2005)

La nouveauté informative peut également provenir du caractère métaphorique des SND (cf. 20 et 21$)$ :

20) Visage carré, cheveux roux carotte, voix rauque, un débit de mitraillette, Muriel Robin s'est installée au forcing en haut de l'affiche. Seule en scène, elle joue les "quadras » hystériques, les mémères à toutous, les bonnes copines, les emmerdeuses... Cette petite bombe vous fait crever de rire. (M.-J. Reichler-Béguelin, 1995, 76)

21) Pour supporter, pour respirer, Sophie Talneau a tenu le journal de sa mise à l'index. C'est drôle et piquant, quotidien et naïf, pertinent et vachard. Au ramdam idéologique, cette Bridget Jones d'ANPE préfère les notations incisives et cruelles. (Libération)

Enfin, tous ces $\mathrm{N}$ sont passibles d'expansions ( $c f .22$ et 23) :

22) Ses premières chansons, il (B. Biolay) les écrit donc en anglais. Pendant deux ans, il court le cacheton. Ce bon élève du Conservatoire qui sait jouer violon, guitare, piano, tuba et trombone à coulisse fait tout. (Libération, 17/12/03)

23) Pour autant, Piège ne se soumet pas aux vieux diktats du luxe. Ce salarié de haut niveau, qui refuse d'en donner le montant (10000 mensuels?), n'abjure ni son époque, ni ses origines. Il se flatte d'appartenir à "la première génération qui a connu les fraises tagada et les carambars ». D’où les serveurs en smoking-blouse, l'autorisation du sans-cravate, les menus-déjeuner à $70 €$. $\mathrm{Ou}$, également, son renvoi dos à dos «de la droite qui fait du populisme et de la gauche qui devient libérale». Pourtant, ce petit-fils d'un agent SNCF adhérent CGT et d'un maquisard du Vercors qui ressemblait à Gabin, jongle avec les 35 heures sans les maudire comme tant d'autres. (Libération, 14/01.05)

\section{I.3. Des relatives à tête démonstrative}

Un dernier type d'expressions, peu présent dans les inventaires ${ }^{6}$, est constitué par les relatives en celui qui dont le statut et notamment le degré d'autonomie référentielle, mériterait d'être sérieusement étudié. Du point de vue informatif qui nous préoccupe ici, ce sont les expressions les plus prédicatives. En effet, la part

6. Pour des raisons qui restent à expliquer. 
d'information " connue » se réduit au pronom celui (qui est lui-même ambigu à cet égard, ainsi que l'a démontré G. Kleiber, 1994). D’autre part, la prédication se réalise, de manière plus « canonique » peut-être, et plus explicite certainement, par le verbe de la relative. Enfin, les possibilités d'y adjoindre de l'information sont théoriquement infinies tant syntaxiquement que sémantiquement. Certains auteurs d'ailleurs ne se privent pas d'exploiter fort judicieusement ce potentiel (cf. 25) :

24) Pas de Jackson Richardson. «En fait, témoigne celui qui dirigea la formation tricolore entre 1990 et 2001, pour une fois, il était prêt cinq minutes avant l'heure. Donc, il s'est allongé. Et il s'est endormi. » La plupart de ses coéquipiers avaient été incapables de fermer l'œil la nuit précédente. Voilà comment naît une légende, celle qui poussa son ancien partenaire Philippe Gardent à dire de celui qui donne parfois rendez-vous aux médias sur une aire d'autoroute : " Jackson se couche à la fois tard ou tôt, comme on veut. » (Libération, 21/01/2005)

25) Ce dernier (Yo-Yo Ma) lui (Erwan Fauré) fit même, un jour de juin 1991, la surprise de venir de Boston jouer pour lui, à Pleyel, le Concerto de Schumann. Le virtuose tenait à saluer celui qui, atteint par la limite d'âge, devait d'un coup quitter le Conservatoire national supérieur de musique de Paris et sa place de violoncelle solo à l'Orchestre national d'île-deFrance, qu'il avait contribué à créer, en 1974. (Le Monde, 26/06/03)

\section{I.4. Premier bilan}

Les moyens de " prédiquer » via les SND sont donc nombreux, très diversifiés du point de vue formel - plus que ne le sont les SN définis - et, enfin, très riches en termes de substance informative. En outre, leur richesse donne précisément à penser que la « prédication » s'exerce sans limite, qu'il est pratiquement possible de faire passer «par la bande » (expression de M.-J. Reichler-Béguelin, 1995) toutes sortes d'informations relatives au référent. De là à dire qu'ils sont libres de toute contrainte, il n'y a qu'un pas...

\section{QUE PEUT-ON « PRÉDIQUER ॥ VIA LE SNDP ?}

Ce pas est franchi par certains auteurs (F. Corblin, 1987, et M.-J. ReichlerBéguelin, art. cit.) alors que d'autres (G. Kleiber, 1984, 1988, entre autres, J.-M. Marandin, 1986) émettent de sérieuses réserves à l'encontre des partisans du «prédicatif tous azimuts »si l'on peut les appeler ainsi ${ }^{7}$. Nous rappelons très succinctement ces positions.

\section{I. La ॥ permissivité " prédicative}

L'absence de contrainte en matière de SNDP est soulignée avec force par certains auteurs, comme M.-J. Reichler-Béguelin qui commente (5) vu plus haut en disant :

[...] dans bien des cas, l'énonciateur se trouve devant une série d'alternatives,

et, dans la mesure où l'identification de l'objet-de-discours visé n'est pas

7. Sans porter aucun jugement de valeur. 
compromise, il est libre de sélectionner et de formater à sa guise l'expression référentielle. » $(1995,54)$

[...] L'exemple < cf. (5) dans notre numérotation $>$, montre bien qu'il n'existe pas de contrainte de cooccurrence stricte entre un «antécédent » donné et une certaine forme de rappel [...]. (art. cit., 58)

Voir aussi plus loin :

[...] nous tenons en tout cas à souligner que la prédication assurée par le biais du SN démonstratif n'est pas, ou du moins n'est pas forcément prédéterminée codiquement. Si tel était le cas, on serait conduit à considérer comme quasi délictueuses les recatégorisations innovantes ou paradoxales et les métaphores d'invention supportées par le SN démonstratif, alors $q^{\prime}$ 'elles font partie du fonctionnement le plus normal de cet outil linguistique. (art. cit., 57) ${ }^{8}$

Ce point de vue a été défendu auparavant par F. Corblin (1987) pour qui le rôle du déterminant démonstratif est primordial dans le mode de saisie référentiel opéré par le SND alors que la tête lexicale ne jouerait, selon lui, pratiquement aucun rôle :

Le groupe nominal Ce $\mathrm{X}$ isole son designatum en vertu de la proximité et, quel que soit son contenu, classifie ce designatum comme X. (F. Corblin, 1987, 209, cité par G. Kleiber, 1988 : 52, les gras sont de nous)

Dans cette optique, l'opération de reclassification est, selon l'auteur :

[...] une opération interprétative régulièrement associée à l'usage du démonstratif, et théoriquement "libre"; une succession quelconque un $\mathrm{Ni}$... ce $\mathrm{Nj}$ est régulièrement interprétable comme désignation de $\mathrm{Ni}$ reclassifié comme $N j$, et il paraît très difficile de circonscrire, sur des bases strictement linguistiques, un ensemble de reclassifications qui seraient prohibées. Les reclassifications peuvent seulement être évaluées, linguistiquement, comme mise en rapport de référence virtuelle: 1) ce $N j$ désigne l'objet $x$ précédemment construit comme $\mathrm{Ni}$; 2) il opère la reclassification d'un $\mathrm{Ni}$ comme $\mathrm{Nj}$ (F. Corblin, 1987, 212 ; nous soulignons)

Avant d'envisager une position alternative à celles-ci, nous ferons trois observations. La première, déjà énoncée en introduction, renvoie aux divergences liées à la recevabilité des énoncés comportant un SNDP. Nous avons, peut-être à tort, du mal à accepter des énoncés comme ${ }^{9}$ :

26) Deux arbres encadraient l'entrée et ces sentinelles dormaient (F. Corblin, 1987, 211)

27) La mer était constellée de voiles, et ces papillons semblaient immobiles (F. Corblin, 1987, 211)

En outre, bien des exemples de reclassification proposés par F. Corblin font suite à un contexte gauche minimaliste, donné entre parenthèses, sans que le SNDP soit suivi d'une quelconque prédication (cf. op. cit. 210, 211, 213, entre autres). Tels quels, les enchaînements ne posent pas trop de problème, si ce

\section{Voir aussi, p. 59.}

9. W. De Mulder nous suggère (cp) à juste titre que la coordination est en pour partie responsable. 
n'est une interrogation sur le degré de leur pertinence dans l'enchaînement. En d'autres termes, on ne peut s'empêcher de se demander pour quelle raison le locuteur éprouve le besoin de reclassifier le référent:

28) (Jean est venu.) Ce professeur de philosophie...

Ce type toujours optimiste... (F. Corblin, 1987, 213, son ex. 30)

Ensuite, il suffit de compléter l'énoncé pour se rendre compte que l'acceptabilité des SNDP est très liée au cotexte, comme le suggère le contraste entre (29), (31), d'une part et, d'autre part (30) et (32) :

29) ?(Jean est venu.) Ce professeur de philosophie, pour une fois, m'a offert des fleurs.

30) (Jean est venu.) Ce professeur de philosophie, pour une fois, ne nous a pas bassinés toute la soirée avec la métaphysique de Kant.

31) ?(Jean est venu.) Ce type toujours optimiste travaille dans l'informatique.

32) (Jean est venu.) Ce type toujours optimiste se révèle, en toute occasion, un convive très agréable.

Ce que F. Corblin ne conteste d'ailleurs pas, puisqu'il précise :

Il est assez probable qu'en fonction des références virtuelles impliquées, des mécanismes sémantiques ultérieurs complètent ces reclassifications et les évaluent selon le degré de motivation qu'il est possible de leur associer dans un contexte.

(F. Corblin, 1987, 212, nous soulignons)

\subsection{Trois limites à la prédication via le SNDP}

Cette conception peu regardante sur les contenus prédicatifs des SND est discutée par G. Kleiber (1984, 1988, entre autres) et J.-M. Marandin (1986). Trois contraintes ressortent de ces travaux.

\subsection{Une relation attributive entre les $\mathrm{N}$ des SNDP et de sa source}

La première fait intervenir la nécessité d'une relation attributive entre le SND et son antécédent. Celle-ci s'exprime diversement selon les auteurs. Elle est très stricte et exigeante sur le rapport entre la source et le $\mathrm{N}$ du SND chez J.M. Marandin (1986) ${ }^{10}$ et rigoureusement conditionnée par le sémantisme du $\mathrm{N}^{11}$ :

L'interprétation anaphorique d'un SN démonstratif avec pour nom recteur certains substantifs, requiert l'occurrence effective dans le contexte gauche d'un terme avec lequel ils peuvent être mis en relation par une relation « êtreX». (J.-M. Marandin, 1986, 77) $)^{12}$

Pour G. Kleiber (1984, 1988), elle provient du sens-même du démonstratif :

10. C'est aussi mutatis mutandis la position de A. Maes et Noordman.

11. Voir les discussions menées par G. Kleiber (1988) et W. De Mulder (1997) à ce propos.

12. Qui exclut la relation d'identité du type : «J'ai rencontré madame Onassis. Cette Jackie Kennedy prenait l'avion pour Londres. ». 
Une description démonstrative $c e N$, anaphorique ou non, équivaut à une structure attributive classificatoire présupposée du type Ce $N$ est un/du $N$ (ou $N$ ) (G. Kleiber, 1984 ; 1988, 62)

Avec, pour conséquence, que :

Le rôle crucial du nom recteur est donc de limiter le domaine des référents possibles à ceux qui admettent d'être nommés ou classés comme des N. (ibid.)

Elle est en outre, de l'aveu même de l'auteur, et par contraste avec celle de J.-M. Marandin (1986) :

[...] beaucoup moins stricte en ce qu'elle n'exige pas une paraphrase correspondant à une phrase copulative mettant en relation le terme anaphorique et un élément du cotexte. Le rapport attributif postulé ne s'établit pas entre un élément du contexte (ou le contexte dans sa globalité) et la description anaphorique mais entre le référent conçu comme non classifié ou non nommé et le substantif recteur. (G. Kleiber, $1988: 63$ )

Cette contrainte présente un double avantage. Elle permet de faire le départ entre les SNDP liés sémantiquement à leur source et ceux qui ne le sont pas. D'autre part, elle rend compte des différences entre (33) et (34) et notamment du rôle de la hiérarchie-être qui s'applique dans (33) et mais pas dans (34) à propos duquel G. Kleiber explique que :

Les traits analytiques et stéréotypiques de livre ne comportent pas le trait être un roman qui serait nécessaire pour opérer une saisie référentielle anaphorique : un livre peut être un roman et c'est tout [...]. (G. Kleiber, 1988, 64)

33) Il y avait sur la table un roman. Ce livre... (G. Kleiber, 1988, 65)

33a) Le x identifié comme un roman est un livre

34) (?) Il y avait sur la table un livre. Ce roman... (F. Corblin, 1987, 210, l'indexation est de G. Kleiber, 1988, 62)

34a) Le $x$ identifié comme un livre est un roman

Dans cette relation attributive, le $\mathrm{N}$ de la source joue un rôle crucial, comme le montre la citation ci-dessus, en limitant, lorsque le SN est indéfini, l'apport de connaissance délivré par le $\mathrm{N}$ aux traits et aux stéréotypes qui lui sont associés. Cette contrainte rend compte de la bizarrerie de (5) où le sens de dame ne comporte pas stéréotypiquement les traits de bourgeoise et a fortiori encore moins ceux de mal attifée :

? le $\mathrm{x}$ identifié comme une dame est une bourgeoise mal attifée

\subsubsection{Une contrainte cotextuelle}

Quand manifestement l'information n'est pas filtrée par le N, c'est au cotexte de justifier la prédication :

Notre proposition [...] prédit aussi l'impossibilité d'un enchaînement un $\mathrm{Ni}$ (hyperonyme)... Ce $\mathrm{Nj}$ (hyponyme) si rien dans le cotexte gauche ne justifie cette restriction. (G. Kleiber, 1988, 64)

Il doit le faire dans le cas de relations non conventionnelles entre le SNDP et sa source, telle qu'établie par ce torchon dans (4) où l'anomalie provient, comme 
l'explique G. Kleiber (1988, 65), de ce qu'il manque l'assertion métaphorique intermédiaire comme quoi les livres sont des torchons:

4) Il y avait sur la table un livre. Ce torchon... (F. Corblin, 1987, 210)

4a) ? Le $x$ identifié comme un livre est un torchon

De là l'idée qu'en l'absence de relations sémantiques :

les reprises, dit G. Kleiber, sont plus libres mais obéissent toujours à la même contrainte générale : l'interlocuteur doit pouvoir s'expliquer les raisons de la classification comme $N$ du référent visé. (G. Kleiber, 1988, 66)

On comprend dès lors pourquoi (1) pose problème. D'une part, il ne préexiste pas de relation sémantique entre chasseur et allemand, qui n'est en rien un trait prototypique du $\mathrm{N}$ de chasseur. D'autre part, rien dans l'environnement de la p1 ne fournit de solution à ce problème classificatoire :

? Le $x$ identifié comme un chasseur est (un) Allemand

\subsubsection{Le mode de donation défini vs indéfini de la source}

La troisième contrainte mise au jour par G. Kleiber $(1988,2001)$ et G. Kleiber, R. Patry et N. Ménard (1990, 134-135) provient du mode de donation qu'exercerait le référent-source sur la recevabilité des SNDP. En effet, ceux-ci passent mieux en présence d'une source définie :

Le problème mérite d'être étendu aux antécédents hyperonymiques définis. Nos informateurs ont trouvé < (35) [dans notre numérotation] > moins anormal que les énoncés à hyperonymes indéfinis mais ont encore noté que la prédication hyponymique de livre par roman leur paraissait injustifiée :

35) Il y a sur la table le dernier livre de Françoise Sagan. Ce roman raconte...

Le nom propre apparemment faciliterait aussi les choses :

Avec un nom propre comme antécédent, les choses sont plus faciles comme en témoignent des exemples classiques tels que $<(36)$ [dans notre numérotation] $>$ :

36) À 60 ans, Paul Dubois se retira dans son village. Cet ancien coureur cycliste ...

Cette contrainte s'illustre également par le contraste entre (37) et (38) :

37) *Paul est allé voir un avocat. Cet avocat de 35 ans ... (G. Kleiber, 2001, 187, son ex. 11)

38) Paul est allé voir l'avocat qui habite dans la vieille ville. Cet avocat de 35 ans ... (G. Kleiber, 2001, 188, son ex. 13)

La différence, explique l'auteur, réside dans la présentation du référent de l'expression antécédent. Quand il est présenté au moyen d'une expression définie (ou terme singulier tel que nom propre, description définie, description démonstrative, etc.), il apparaît comme un référent connu autrement que par sa seule appartenance à la classe des $N$, de telle sorte que l'information (nouvelle ou non pour l'interlocuteur) peut être acceptée comme étant en somme une information disponible ou inférable à partir de la présentation définie (ou particulière) faite du référent. Si, au contraire, il s'agit d'un antécédent indéfini, comme dans le cas de un avocat (...) la seule connaissance que nous en ayons est le fait de son appartenance à la classe des $N$. Les seules inférences auxquelles donne lieu une telle présentation sont alors celles qui sont liées à son appartenance à la classe des 
avocats (...), c'est-à-dire (...) celles qui découlent d'une connaissance prototypique ou stéréotypique ou encore d'une connaissance par défaut des membres de la classe N concernée. (G. Kleiber, 2001, 187-188, nous soulignons).

Ce point est capital car il invite à dissocier les énoncés selon leur source, définie ou non. Il permet également de comprendre la gradation établie dans les énoncés ci-dessus. La reprise par ce roman est envisageable dans (35), parce que l'on sait que F. Sagan est romancière et que sa production écrite se constitue pour l'essentiel de romans. De même, le fait de savoir que P. Dubois a été coureur cycliste (cf. (36)) est lié aux connaissances extra-linguistiques que l'on a (ou non) du référent ainsi dénommé. Est-ce à dire qu'en présence de sources définies, il n'y a plus rien qui bride les SNDP ? Rien n'est moins sûr car il reste à expliquer pourquoi l'enchaînement (39) ainsi complété, passe mal :

39) ? À 60 ans, Paul Dubois se retira dans son village. Cet ancien coureur cycliste adore les andouillettes.

Ceci étant, cette contrainte éclaire d'une lumière nouvelle certaines illustrations exploitées par les partisans des SNDP «tout venants", dans la mesure où beaucoup de leurs exemples exploitent des séquences anaphoriques dont la source est définie, ce qu'illustrent quelques exemples repris de F. Corblin (op. cit.) :

40) Les ouvriers considérèrent le plancher $\mathbf{d u}$ wagon et décidèrent que pour une nuit, on pouvait se satisfaire de ce lit. (F. Corblin, 212, son ex. 27)

41) (La jeune fille était charmante) mais il fut impossible de faire sourire ce glaçon. (F. Corblin, 213, son ex. 32)

42) (Pierre tint bon) et ce roc ne bougea pas d'un pouce. (F. Corblin, 213, son ex. 33)

\subsection{Bilan}

Force est de conclure, au terme de cette deuxième partie, qu'en dépit de leur apparente liberté, les SNDP obéissent à des contraintes :

- lexicales qui les lient, par la relation attributive être (un/du) $N$, à leur source lorsqu'ils lui sont sémantiquement rattachés ;

- contextuelles, puisqu'il s'est avéré que le mode de donation de leur source joue un rôle central ainsi que, comme cela est quasi unanimement suggéré, le cotexte d'accueil des segments coréférentiels.

Une fois ces contraintes posées, il reste à déterminer s'il n'y a pas moyen d'introduire, malgré un SN indéfini, des contenus moins "codifiés » et si, avec une source définie, il n'y a pas matière à préciser le rôle que joue le cotexte.

\section{OUVERTURES ET FERMETURES}

En réponse à cette question, nous fournirons des éléments de réponse distincts selon le mode de donation du SN-source. 


\section{I. La source est introduite via un $\mathbf{N}$}

Dans l'hypothèse où, en présence d'une source indéfinie, les contenus du SNDP sont limités aux informations stéréotypées liées au $N$ du $\mathrm{SN}$, on peut faire l'hypothèse que les possibilités de prédication via le SN démonstratif peuvent être envisagées sous deux conditions au moins.

\section{I.I. La mention dans le cotexte d'une "source de savoir »}

Soit l'exemple (43) où la source du SNDP est un SN indéfini :

43) ? Un homme entra dans un bar. (Ce molosse barbu + cet italien bilingue + cet intellectuel de gauche + ce fan de David Bowie + ce notaire + ce Breton) se dirigea d'un pas décidé vers le comptoir

Rien dans les environnements gauche et droit du SNDP ne motive les apports informatifs entre parenthèses. Il suffit, en revanche, d'introduire dans le cotexte une "source de savoir», réelle ou potentielle, pour que certaines informations passent beaucoup mieux :

44) Un homme entra dans un bar. Le patron dévisagea avec curiosité (ce molosse barbu $+{ }^{*}$ cet italien bilingue).

45) Je venais de m'installer à un table lorsqu'un homme entra dans le bar. (Ce barbu/molosse/ivrogne/vieillard) se dirigea d'un pas hésitant vers le comptoir.

De fait, l'introduction d'une sorte de « sujet de conscience » dans le cotexte, le patron du bar, dans (44), ou un sujet-énonciateur à la première personne, dans (45), rend possible l'introduction d'informations non conventionnelles sur le référent ${ }^{13}$. Maintenant, les énoncés ci-dessus montrent que ce principe ne permet pas tout.

\section{I.2. Nature et accessibilité des informations}

Encore faut-il prendre en compte la nature des informations accessibles à ce sujet de conscience dans la situation. Dans (44) et (45), les SNDP qui passent sont celles qui diffusent des informations accessibles par la vue - en l'occurrence la taille, la tranche d'âge - qui sont, par défaut, les informations disponibles dans la plupart des situations mettant en présence des individus. Dans (46), le fait que le personnage passe sa commande rend plausible le SNDP cet

13. M.-J. Reichler-Béguelin tire argument de la reprise hyponymique de l'exemple suivant: "C'est ainsi qu'au Rivier (domaine de la présidente) de magnifiques oiseaux blancs ont pu être observés pendant 15 jours. Ils étaient très grands, très nombreux, dotés d'un long et gros bec jaune-rouge qu'ils glissaient dans l'eau pour pêcher, en avançant à pied dans la vasière. Ces "spatules" ont pu être vues par tous ceux qui ont emprunté les promenades piétonnières organisées pour le public » (nous soulignons), pour réfuter la contrainte posée par G. Kleiber et al. (1990) sur le SN indéfini, en disant (note 37, p. 73) : "Il est bien possible qu'une étude empirique permette de confirmer cette intuition, mais l'exemple qu'on vient d'analyser montre bien que le rappel spécifiant concerne aussi des objets-de-discours introduits sous forme indéfinie ». Or, si la source est effectivement un SN indéfini, son référent est appréhendé par des sujets de conscience ( $c f$. les verbes en italiques dans l'exemple), ce que l'auteur souligne sans établir la corrélation. 
italien bilingue, puisqu'en parlant, il rend disponibles des informations sur un éventuel accent, donc la nationalité, etc. :

46) Un homme entra dans un bar et commanda une Budweiser au comptoir. Le patron dévisagea avec curiosité (ce molosse barbu + cet italien bilingue).

En revanche, les reprises de (47) et (48) restent peu plausibles car les informations délivrées par les SNDP ne sont pas, sinon peu, accessibles étant donné les circonstances dénotées par le cotexte. Seule, l'intervention d'un "narrateur omniscient », susceptible d'anticiper la suite des événements, justifie a fortiori la prédication d'informations sans cela inaccessibles ${ }^{14}$ :

47) ? Un homme entra dans un bar et commanda une Budweiser au comptoir. Le patron dévisagea (cet intellectuel de gauche + ce fan de David Bowie + ce représentant de commerce). Ce Charentais se dirigea d'un pas décidé vers le comptoir.

48) Un homme entra dans un bar et commanda une Budweiser au comptoir. Cet intellectuel de gauche allait bientôt convaincre le patron de voter contre la constitution européenne.

\subsection{La source est introduite au moyen de SN définis}

Lorsque la source des SNDP est introduite par un SN défini, la connaissance du référent étant acquise - ou susceptible de l'être -, suivant la suggestion de G. Kleiber, par d'autres voies que le cotexte, les formes de SNDP semblent effectivement se soustraire à toute forme de contrainte. C'est, du reste, ce qui explique que, dans les portraits journalistiques exploités pour cette étude, les rédacteurs ne se privent pas de diffuser "par la bande » toutes sortes d'informations y compris les plus « exotiques » sans qu'il y ait rien à y redire :

49) Voici Piège, revêtu de sa propre casaque, lancé dans la course aux étoiles. Car les palaces viennent de réaliser que la cotation Michelin est cruciale en ces temps de clientèle nomade. Le Georges- $\mathrm{V}$ et le Plaza affichent trois macarons. Le Bristol, deux. Le Crillon n'en a qu'un et se doit de monter en gamme. Pour autant, Piège ne se soumet pas aux vieux diktats du luxe. Ce salarié de haut niveau, qui refuse d'en donner le montant (10 $000 €$ mensuels ?), n'abjure ni son époque, ni ses origines. Il se flatte d'appartenir à « la première génération qui a connu les fraises tagada et les carambars ». D'où les serveurs en smoking-blouse, l'autorisation du sans-cravate, les menusdéjeuner à $70 €$. Ou, également, son renvoi dos à dos « de la droite qui fait $d u$ populisme et de la gauche qui devient libérale». Pourtant, ce petit-fils d'un agent SNCF adhérent CGT et d'un maquisard du Vercors qui ressemblait à Gabin, jongle avec les 35 heures sans les maudire comme tant d'autres. Et il a fait campagne auprès de ses commis pour qu'ils aillent voter contre Le Pen, en 2002. Pas baptisé, il n'est pas franc (Libération, 14/01/05)

\footnotetext{
14. C'est ce que suggère, du reste, M.-J. Reichler-Béguelin : «les anaphores de cette catégorie sont parfois le support de subtils faits de polyphonie, quand la reclassification de l'objet, n'est plausible qu'à condition d'être assignée au point de vue d'un personnage, en fonction de ce que l'on sait de lui. [...] Il ne fait pas de doute que la recevabilité des anaphores démonstratives est en partie fonction de ces phénomènes » $(1995,75)$.
} 
50) Marc Jacobs, 41 ans, Américain, styliste de sa marque et directeur artistique chez Vuitton, qu'il a contribué à relancer. Doué en affaires, il se pose plutôt en rebelle du luxe.

\section{Double étiquette}

Il y a des préoccupations plus angoissantes, mais lui non plus ne sait jamais quoi se mettre quand il sort, redoute le geste absurde de se déshabiller pour se rhabiller, ne va pas seul à des fêtes, et surtout pas à des soirées où il ne connaît personne. Mais c'est improbable. Le soir du nouvel an, il en a profité " pour travailler » et s'est nourri d'un « sandwich au poulet », mangé sur le canapé, chez lui, devant la télé. Plus tard, à l'aube, il est sorti, doit-on à la vérité. Bref, cet homme qui porte un pull peluché bleu marine Comme des garçons et des sneakers blanches est exactement comme vous. (Libération, 04 mars 2005)

Cela ne va pas sans provoquer quelques couacs - c'est le cas de le dire avec, dans (51), le SN ce virtuose de l'accordéon :

\section{Daniel Libeskind \\ Bâtisseur d'espoir}

Fils d'immigrés juifs polonais, cet architecte a remporté le concours pour la reconstruction du World Trade Center.

Né en Pologne après la Seconde Guerre Mondiale, Daniel Libeskind y a vécu l'antisémitisme du régime communiste après que ses parents avaient subi celui des nazis. Sa famille a rapidement émigré en Israël, où elle s'est vite lassée de la vie dans les kibboutzim avant de partir pour les États Unis. De quoi donner le goût du nomadisme - avec quatorze déménagements en trente-cinq ans! - à ce virtuose de l'accordéon! (TV Hebdo, 2005)

Apparemment ce n'est pas en soi le contenu informatif qui est en cause, tout aussi « pointu » à cet égard qu'il l'est en (49) et (50). L'hypothèse que l'on peut formuler pour rendre compte de cette incongruité tient précisément à la nature prédicative du SN démonstratif. En effet, dans la mesure où il y a prédication, on peut penser que celle-ci se soumet aux contraintes auxquelles sont assujetties les prédications en général, à savoir le respect d'un principe de cohérence. En temps «normal», celui-ci concerne deux propositions ou segments adjacents (ou hiérarchisés). Mais, en présence d'une anaphore prédicative, ce principe semble naturellement s'instaurer entre le SNDP et la prédication de sa proposition-hôte. Le lien avec le cotexte gauche serait moins important compte tenu du sens du SN démonstratif « qui fait intervenir le contexte d'énonciation de sa propre occurrence » (cf. G. Kleiber, 1988, 68) abstraction faite des circonstances d'évaluation qui en motiveraient l'emploi. Ainsi, l'énoncé (39) vu plus haut se paraphraserait-il comme suit :

52) ? (p1) À 60 ans, Paul Dubois se retira dans son village. (p2) (ce $x$ identifié comme étant Paul Dubois est un ancien coureur cycliste) (et) (p3) (Il) adore les andouillettes.

Or, comme cela a été démontré dans les travaux sur la cohérence textuelle (cf. notamment M. Charolles, 1995), la succession de deux propositions invite l'interprète à établir entre celles-ci un lien, le plus «naturel » - et le plus fort étant celui de causalité, le plus faible étant un lien d'analogie. Dans le cas des SNDP, la prédication est, si l'on peut dire, « encapsulée » dans la proposition. 
En d'autres termes, les deux prédications seraient plus intimement liées qu'elles ne le sont en (52). De sorte que l'on peut se demander si cela n'oblige pas à établir le lien de cohérence le plus fort entre les deux, un lien de causalité donc. En effet, dans (49), on comprend que c'est parce que Piège est petit-fils d'un adhérent CGT et d'un maquisard, donc plutôt " humaniste de gauche », qu'il jongle avec les 35 heures ou, dans (50), que c'est parce qu'il porte les habits de monsieur-tout-le-monde (et non pas ceux de la marque pour laquelle il travaille entre autres) que $\mathrm{M}$. Jacobs est comme le lecteur de l'article :

49a) Pourtant, ce petit-fils d'un agent SNCF adhérent CGT et d'un maquisard du Vercors qui ressemblait à Gabin, jongle avec les 35 heures sans les maudire comme tant d'autres.

50a) Bref, cet homme qui porte un pull peluché bleu marine Comme des garçons et des sneakers blanches est exactement comme vous.

Le rapport est en revanche nettement plus difficile (du moins pour nous) à construire entre le goût du nomadisme et celui de l'accordéon.

\section{CONCLUSION}

Partie de l'observation que les SNDP sont formellement et informationnellement très diversifiés et utilisés très librement en apparence, nous avons fait valoir deux types de contraintes à même d'expliquer certains cas d'irrecevabilité et de motiver l'usage de ces unités.

La première, suggérée par les travaux de G. Kleiber, fait intervenir le mode de donation de la source du SNDP. Il est ainsi apparu que les SN indéfinis limitent les informations référentielles aux stéréotypes délivrés par le N. Dès lors, toute autre forme de connaissance doit être motivée dans le cotexte, par exemple par l'introduction de sujets de conscience dans des situations qui leur donnent effectivement accès à ces connaissances.

La seconde contrainte provient du caractère prédicatif des SNDP qui, nous en faisons l'hypothèse, sont assujettis aux mêmes contraintes de cohérence que les enchaînements prédicatifs inter-propositionnels "standards ", à ceci près que, icôniquement, ils semblent établir des liens plus forts que ceux qui régissent les prédicats apparaissant dans des propositions «séparées ».

Cela étant, les emplois étudiés ici ne constituent qu'une petite partie - et sans doute la moins ardue - des SNDP, dont la teneur informative se révèle souvent plus ambivalente qu'elle ne l'est dans les extraits commentés ici, autant d'ailleurs que celle de leur source...

\section{Bibliographie}

BOtLEY, S. \& MCENERY, Y. (200I), Demonstratives in English, Journal of English Linguistics, 29/I, 7-33.

Charolles, M. (1995), Cohérence, cohésion et pertinence du discours, Travaux de linguistique 29 , |25-|5|.

CORBLIN, F. (1987), Indéfini, défini et démonstratif, Genève-Paris, Droz. 
DE MULDER, W. (1997), Les démonstratifs : des indices de changement de contexte, in N. Flaux, D. Van de Velde et W. De Mulder (éds), Entre général et particulier: les déterminants, Arras, P.U., I37-200.

DE MULDER, W. (200 I), Peut-on définir les SN démonstratifs par leurs contextes, in H. Kronning et al. (éds), Langage et référence. Mélanges offerts à K. Jonasson à l'occasion de ses soixante ans, Uppsala, Acta Universitatis Upsaliensis, II5-123.

KLEIBER, G. (1984), Sur la sémantique des descriptions démonstratives, Lingvisticae Investigationes VIII, I, 63-85.

KLEIBER, G. (1986), Déictiques, embrayeurs, «token reflexives », symboles indexicaux, etc. : comment les définir?, L'information grammaticale, 30, 3-21.

KLEIBER, G. (1986), Pour une explication du paradoxe de la reprise immédiate, Langue française 72 , 54-79.

KLEIBER, G. (1988), Sur l'anaphore démonstrative, in G. Maurand (éd.), Nouvelles recherches en grammaire, Albi, Université Toulouse le Mirail, 5I-74.

KLEIBER, G. (1989), Les démonstratifs de près et de loin, Strasbourg 2, Anaphore \& Deixis 2.

KLEIBER, G. (1994), Anaphores et pronoms, Louvain-la-Neuve, Duculot.

KLEIBER, G. (200 I), L'anaphore associative, Paris, Seuil.

KLeIBER, G. (2003), Adjectifs démonstratifs et point de vue, Cahiers de Praxématique 4I, 33-54.

KLEIBER, G., PATRY, R., MÉNARD, N. (1990), «L'anaphore associative : dans quel sens roule-t-elle?, in C. Schnedecker et al. (éds), L'anaphore associative, Paris, Klincksieck, 129-148.

LANGUE FRANÇAISE I 20 (1998), Les démonstratifs : théories linguistiques et textes littéraires, M.-N. GaryPrieur \& M. Léonard (éds), Paris, Larousse.

LERAT, P. (1988), Anaphore nominale et cohésion discursive : 3 relations d'interdéfinition, in H. Nølke (éd.) Opérateurs syntaxiques et cohésion discursive, Nyt Nordisk Forlag Arnold Busck, I37-I 46.

MAES, A.A. \& NOORDMAN, L.G.M. (1995), Demonstrative nominal anaphors: a case of non identical marketings, Linguistics, 33, 255-282.

MANUÉLIAN, H. (2003), Descriptions définies et démonstratives: analyse de corpus pour la génération de texte, Thèse NR, dir. P. Riley \& J.-M. Pierrel, Nancy 2.

MARANDIN, J.-M. (1986), Ce est un autre. L'interprétation anaphorique du SN démonstratif, Langages $81,75-89$

MILNER, J.-C. (1982), Ordres et raisons de langue, Paris, Seuil.

ReICHLER-BÉGUeLIN, M.-J. (1995), Alternatives et décisions lexicales dans l'emploi des expressions démonstratives, Pratiques 85, 53-87.

ReICHLER-BÉGUeLIN, M.-J. (1998), L'usage des démonstratifs dans les Fables de La Fontaine, Langue Française 120, 95-109.

WIEDERSPIEL, B. (1994), Descriptions démonstratives anaphoriques: interprétations et stratégies référentielles, Thèse de doctorat NR, dir. G. Kleiber, Strasbourg 2.

WiederSPiEL, B. (1995), Sur quelques aspects de la saisie démonstrative, Pratiques 85, I | 3-125. 